\title{
Performance enhancement of an automotive silencer using acoustic metamaterial baffles
}

\author{
Daniel Deery ${ }^{1}$, John Kennedy ${ }^{2}$ \\ Department of Mechanical, Manufacturing and Biomedical Engineering, Trinity College Dublin, \\ Dublin, Ireland \\ ${ }^{2}$ Corresponding author \\ E-mail: ${ }^{1}$ deeryda@tcd.ie, ${ }^{2} j k e n n e d 5 @ t c d . i e$
}

Received 12 April 2021; received in revised form 21 April 2021; accepted 29 April 2021 DOI https://doi.org/10.21595/vp.2021.21989

Check for updates

Copyright (C) 2021 Daniel Deery, et al. This is an open access article distributed under the Creative Commons Attribution License, which permits unrestricted use, distribution, and reproduction in any medium, provided the original work is properly cited.

\begin{abstract}
The potential for acoustic metamaterials to provide a breakthrough in sound attenuation has been unlocked in recent times due to advancements in additive manufacturing techniques. These materials allow the targeting of specific frequencies for sound attenuation. To date acoustic metamaterials have not been experimentally tested in an automotive silencer for performance enhancement. This study investigates the effect of acoustic metamaterials within a representative automotive silencer. These results are then compared with commonly used techniques in the silencer industry to gauge the effectiveness of the acoustic metamaterials. COMSOL simulations were used to validate the developed test rig and were compared to experimental results which were obtained using the two-load transmission loss test method. Through this testing method, the implementation of the acoustic labyrinthine DENORMS cylinder proved to be the most significant improvement in transmission loss within the silencer, with an increase in transmission loss of $40 \mathrm{~dB}$ at $1500 \mathrm{~Hz}$. It was also found that the traditional hard wall internal baffle silencer performance can be improved by replacing it with a DENORMS internal baffle. Acoustic metamaterials have potential as a novel method for automotive companies to attenuate sound of specific frequencies.
\end{abstract}

Keywords: acoustic metamaterials, automotive silencer, comsol, two-load test rig, labyrinthine, cylinders, baffles.

\section{Introduction}

In an automotive exhaust system, the silencer is used to reduce the noise output to the surrounding environment created by the engine. A petrol engine with no silencer radiates exhaust noise between 90 and $100 \mathrm{~dB}(\mathrm{~A})$, while a diesel engine with no silencer, under identical conditions, radiates exhaust noise in the range from 100 to $125 \mathrm{~dB}(\mathrm{~A})$ [1]. Without a silencer, these noise levels would cause health issues in both humans and wildlife. According to the World Health Organisation (WHO) these health issues include: cardiovascular disease, cognitive impairment, sleep disturbance, reduced performance, tinnitus and aggression [2]. With an increase in the number of automobiles in our cities, it is imperative for the health of the global population to improve silencer performance to help reduce urban noise. The parameter used to measure the performance of the silencer in this paper is the transmission loss (TL) value. Transmission loss determines the sound attenuation properties of the silencer. The transmission loss is the difference in the sound power level between the incident wave entering and the transmitted wave exiting the silencer when the silencer termination is anechoic [3].

The pressure waves in the engine escape into the exhaust system when the exhaust valves open and close repeatedly. The frequency of these sound waves produced by the engine mainly depend on the rpm [4]. A silencer must be able to reduce the noise output of the engine, while trying to minimise the restriction on the flow of exhaust gas. There are two main types of silencers, reactive and absorptive, with most commercial silencers using a mixture of the two [4]. Absorptive silencers rely on fact that sound waves lose energy as they travel through a porous medium, the sound energy is dissipated in the form of heat. Reactive silencers utilise destructive interference 
to attenuate sound. This is achieved whenever the maxima of sound waves that are out of phase by $180^{\circ}$ collide with one another. In a silencer, this can occur whenever there is a change in geometry. The geometric changes investigated in this paper include: expansion and contraction chambers, side inlet and outlet, baffles and internal cylinders.

Acoustic metamaterials are materials with artificial periodic structures which are much smaller than the wavelengths at which they operate, the smallest unit being called the unit cell [5]. These unit cells make up the structure of the acoustic metamaterial baffle (AMB) and acoustic metamaterial cylinder (AMC) and provide the basis of the sound attenuation behaviour in the baffle. The unit cells act as resonators with a high number of degrees of freedom due to the chain length. When sound waves propagate in this structure, due to the modulation of the structure, the propagation of sound waves is hindered, thereby achieving a sound absorption [6]. Using 3D printing machines, these complex internal structures can be created. The metamaterials used in this paper is the DENORMS (Designs for Noise Reducing Materials and Structures) benchmark design. This consists of a periodic unit cell structure of cubes with spherical internal cavities connected through cylindrical openings on each face of the cube [7].

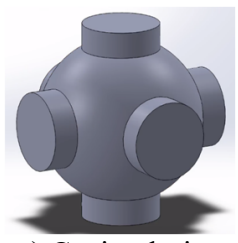

a) Cavity design

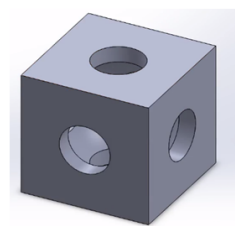

b) Unit cell

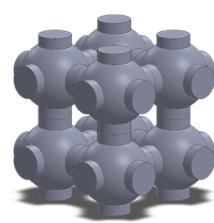

c) Basic design

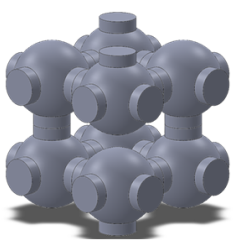

d) Labyrinthine design

Fig. 1. DENORMS metamaterial designs

To create the labyrinthine structure shown in (Fig. 1(d)), the DENORMS benchmark cavity design is altered. This is achieved by removing the cylindrical outlets of the benchmark design to increase the length of the chain of unit cells within the metamaterial. For this application, the DENORMS design was altered to increase the length of the internal chain from four to eight while maintaining the total thickness. The absorption characteristics of the DENORMS cell depend on the number of continuously connected cells in the structure. Increasing the number of cells in a chain moves the peak absorption value to a lower frequency. The absorption characteristics of chains of differing lengths were reported in [8]. This information was used to design the DENORMS metamaterial baffles and cylinders used in this study.

\section{Methodology}

A representative silencer was designed, and the silencer performance was analysed numerically and experimentally. The silencer was first modelled in COMSOL Multiphysics. The simulations were set up to enable easy removal/addition of internal geometries to match the modular nature of the experimental test rig.

The simulations calculated the transmission loss between $300-3500 \mathrm{~Hz}$ with a resolution of $5 \mathrm{~Hz}$. This frequency range is representative of a typical automotive engine output and corresponded to the range in which the experimental rig operates effectively.

The experimental rig used to calculate the silencer performance was the two-load test rig setup. This set up complies with the ASTM standard E2611 for the determination of the properties in an impedance tube [9]. The parameter used to represent the silencer performance was the transmission loss, which can be given by the equation:

$$
T L=10 \log _{10}\left|\frac{W_{i}}{W_{r}}\right| \mathrm{dB},
$$

where $W_{i}$ is the strength of the incident wave and $W_{r}$ is the strength of the reflected wave [10]. 


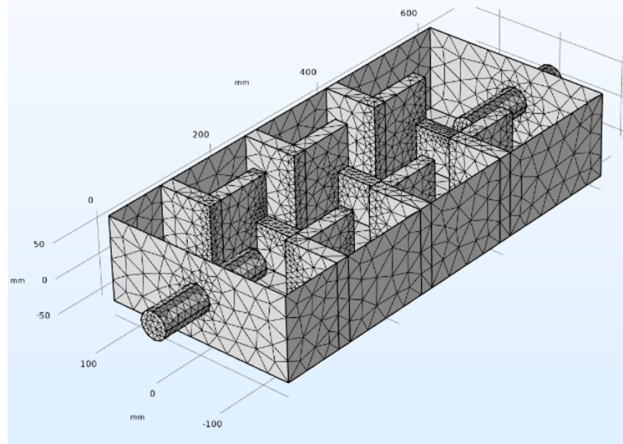

a) Straight inlet

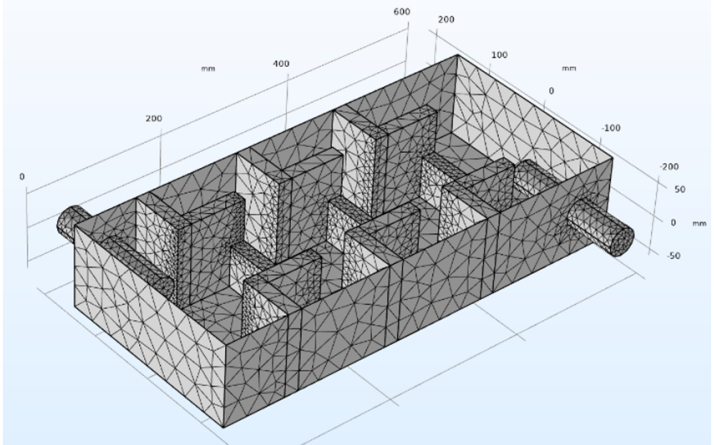

b) Side inlet

Fig. 2. COMSOL simulation set up

In the two-load method there are four waves, two incident and two reflected. Two different loads are used as end conditions, as with only one end condition there are four unknowns and only two equations to solve them. The two-load method is based off the transfer mix approach. Any acoustic element can be modelled off its four-pole parameters:

$\left[\begin{array}{l}p_{1} \\ v_{1}\end{array}\right]=\left[\begin{array}{ll}T_{11} & T_{12} \\ T_{21} & T_{22}\end{array}\right]\left[\begin{array}{l}p_{2} \\ v_{2}\end{array}\right]$

where $p$ is the sound pressure amplitude at the inlet $\left(p_{1}\right)$ and outlet $\left(p_{2}\right)$ of the silencer, and $v$ is the velocities amplitude at the inlet $\left(v_{1}\right)$ and outlet $\left(v_{2}\right)$. T $(\omega)$ is the cell transfer matrix of the acoustic system, giving the four pole parameters. By changing the load conditions, the four poles can be easily obtained. The transmission loss can then be expressed in terms of these four poles and the tube areas [13]:

$T L=20 \log _{10}\left\{\left|\frac{1}{2}\left(T_{11}+\frac{T_{12}}{\rho c}+\rho c T_{21}+T_{22}\right)\right|\right\}+10 \log _{10}\left(\frac{S_{i}}{S_{o}}\right)$,

where $S_{i}$ and $S_{o}$ are the silencer inlet and outlet tube areas respectively, $\rho$ is the fluid density and $c$ is the speed of sound in air.

Using the transfer matrix approach, the experimental transmission loss can be obtained and compared with the COMSOL simulation results. These experimental calculations are solved through MATLAB, giving a narrowband and octave band transmission loss vs frequency graphs. Any performance enhancements from the metamaterial components can then clearly identified.

\section{Results}

The tests were broken down into three groups, each testing group involved changing one geometric entity in the test. These geometric changes are the baffle type, the cylinder type and the inlet/outlet configuration. To ensure that the silencer was behaving appropriately during the experiments, COMSOL simulations were carried out for performance validation.

The results shown in (Fig. 3) display that there is good agreement between the theoretical and experimental performance of the silencer. This demonstrates that the two-load test rig method used to experimentally evaluate the silencer performance is suitable.

The next test conducted involved using the cylinder type as the only variable. Internal cylinders are a common method of increasing transmission loss in an automotive silencer, this test was conducted to establish if a metamaterial cylinder could be used to improve the silencer performance.

In (Fig. 4) all the cylinder types behave similarly between 0-1000 Hz. In general, each of the 
cylinder types provide an improvement in transmission loss across the entire frequency range when compared to the silencer with no cylinders. The DENORMS cylinder has a transmission loss spike between $1500-2000 \mathrm{~Hz}$ and gives an improved transmission loss value of over $40 \mathrm{~dB}$. This metamaterial cylinder consists of a labyrinthine DENORMS chain of 8 layers of unit cells and corresponds to the anticipated spike in transmission loss frequency range from [8].

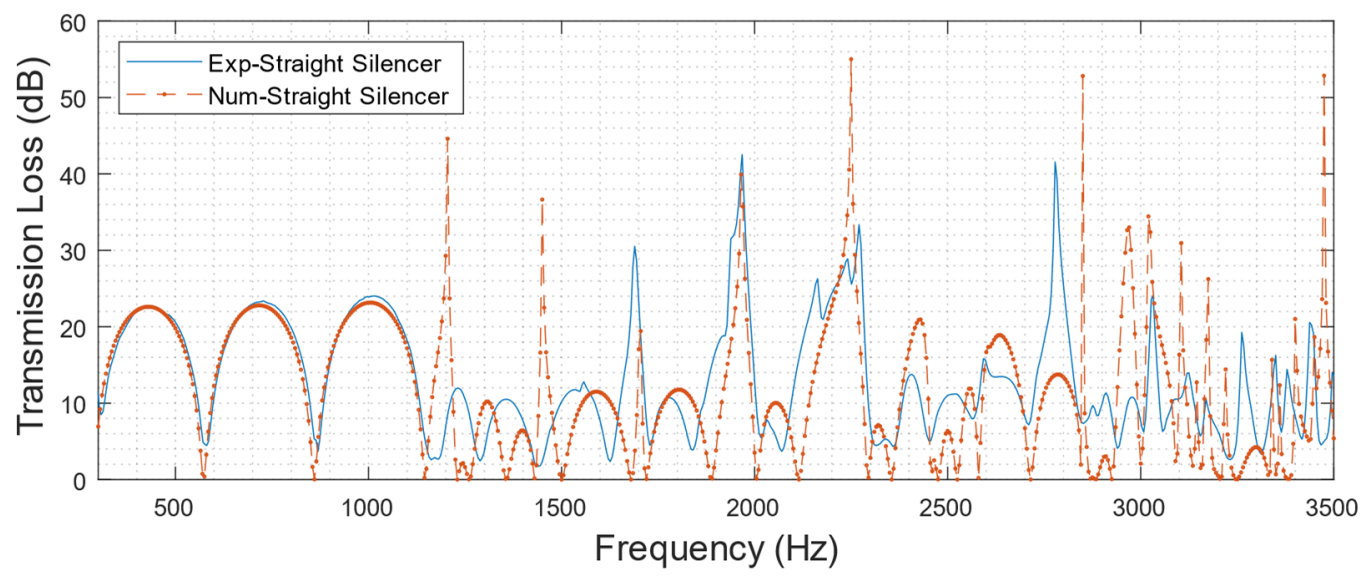

Fig. 3. Basic straight silencer experimental versus numerical results

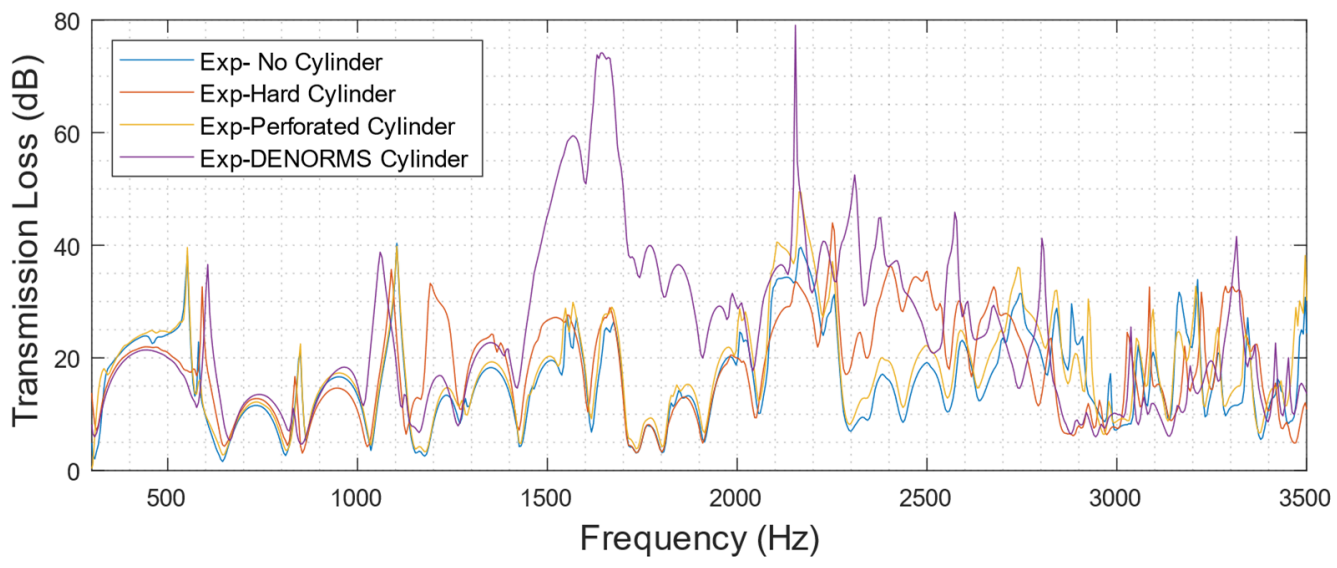

Fig. 4. Cylinder type comparison

The literature suggested that utilising baffles in silencers has been found to improve the transmission loss by more than $50 \%$ [11]. Implementing internal baffles increases the number of internal geometric discontinuities within the silencer thus increases the transmission loss.

The inclusion of the internal baffles increased the performance of the silencer across the entire frequency range. The labyrinthine DENORMS metamaterial baffle improves the performance of the silencer significantly in the 500-1000 Hz frequency range and in the $1500-2000 \mathrm{~Hz}$ frequency range shown in (Fig. 5). The large peak between 500-1000 Hz was an unexpected result and needs to be investigated further. As previously discussed, a peak is expected in the $1500-2000 \mathrm{~Hz}$ which is achieved, confirming that a metamaterial baffle can be used to improve the performance of a silencer. The standard DENORMS metamaterial baffle should increase between $3000-3500 \mathrm{~Hz}$ as it has four-unit cell layers [8]. There is an increase in the transmission loss value of $15 \mathrm{~dB}$ when compared to the hard-internal baffle, illustrated by the yellow spike in this frequency range in (Fig. 5). 


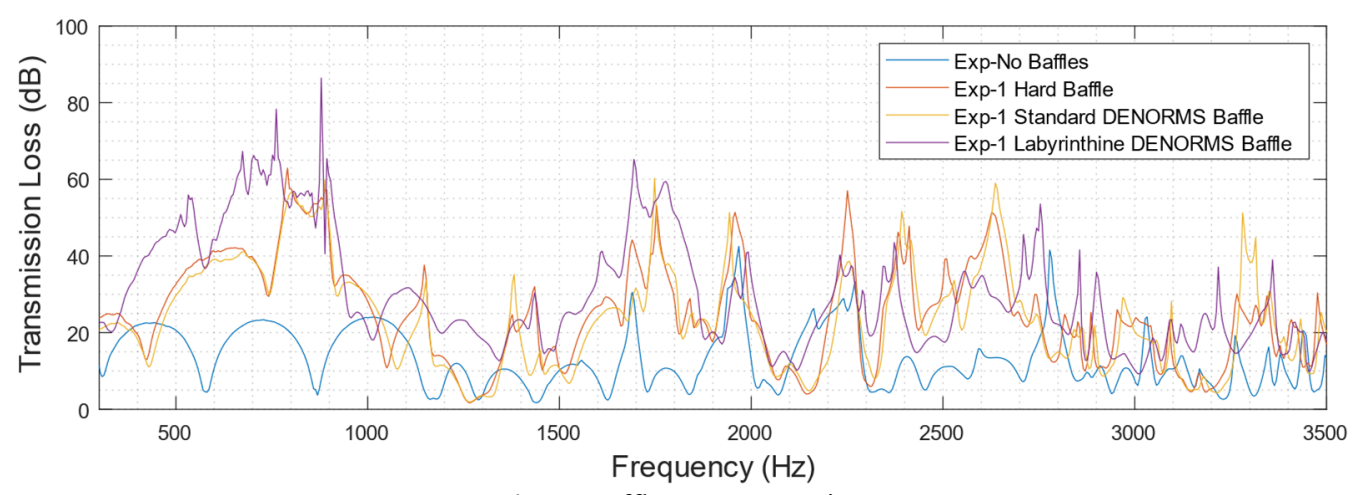

Fig. 5. Baffle type comparison

\section{Conclusions}

This paper investigates the effects acoustic metamaterials have when applied to an automotive silencer. A modular silencer has been successful created to enable experiments to be conducted. Various traditional sound attenuating techniques were investigated and the performance of the silencer has been validated using COMSOL Multiphysics. The side inlet/outlet, internal baffles and internal cylinders all proved to increase the performance of the silencer as they increased the number of interactions between sound waves. These treatments were further enhanced with the implementation of acoustic metamaterials. The application of the acoustic labyrinthine DENORMS cylinder proved to be the most significant improvement in transmission loss within the silencer in this study. The large spike in transmission loss occurred in the expected frequency range and occurred due to the number of unit cell layers within the metamaterial. The standard DENORMS and labyrinthine DENORMS baffles also improved the transmission loss in the appropriate frequency in comparison to the more traditional hard wall baffle. The DENORMS unit cell could be used in the automotive industry to accurately target certain unwanted frequency outputs for attenuation in the exhaust system. The easy modification of the design can be utilized to target different frequency ranges, which increases the applicability of the design.

\section{References}

[1] Lee H. J., Park Y. C., Lee C., Youn D. H. Fast active noise control algorithm for car exhaust noise control. Electronics Letters, Vol. 36, Issue 14, 2000, p. 1250-1251.

[2] Directive 2002/49/EC of the European Parliament and of the Council of 25 June 2002 relating to the assessment and management of environmental noise. Official Journal of the European Communities, 2002.

[3] Hua X., Herrin D. W. Practical considerations when using the two-load method to determine the transmission loss of mufflers and silencers. SAE International Journal of Passenger Cars - Mechanical Systems, Vol. 6, Issue 2, 2013, https://doi.org/10.4271/2013-01-1881.

[4] Potente D. General design principles for an automotive muffler. Proceedings of Acoustics, 2005, https://www.academia.edu/18891822/General_design_principles_for_an_automotive_muffler.

[5] Henríquez V. C., Andersen P. R., Jensen J. S., Juhl P. M., Sánchez Dehesa J. A numerical model of an acoustic metamaterial using the boundary element method including viscous and thermal. Journal of Theoretical and Computational Acoustics, Vol. 25, 2017, p. 1750006.

[6] Kheybari M., Ebrahimi Nejad S. Locally resonant stop band acoustic metamaterial muffler with tuned resonance frequency range. Materials Research Express, Vol. 6, Issue 2, 2018, p. 2.

[7] Dowling L., Flanagan L., Rice H., Trimble D., Kennedy J. The use of a benchmark periodic metamaterial to inform numerical modelling and additive manufacturing approaches. Proceedings of ISMA2018 and USD2018, 2018. 
[8] Kennedy John, Flanagan Lara, Dowling Luke, Bennett G. J., Rice Henry, Trimble Daniel The influence of additive manufacturing processes on the performance of a periodic acoustic metamaterial. International Journal of Polymer Science, Vol. 2019, 2019, p. 7029143.

[9] ISO/AWI 10534-2. Acoustics - Determination of properties in impedance tubes - Part 2: 2-microphone technique for normal sound absorption coefficient and normal surface impedance, https://www.iso.org/cms/render/live/en/sites/isoorg/contents/data/standard/08/12/81294.html.

[10] Seybert A. F., Ross D. F. Experimental determination of acoustic properties using a two- microphone random excitation technique. The Journal of the Acoustical Society of America, Vol. 61, 1977, p. 1362-1370.

[11] Done V., Venkatesham B., Tamma B., Soni K., Dey S., Angadi S., Vishal G. P. Muffler design for a refrigerator compressor. International Compressor Engineering Conference, 2014. 